the pairs of distinct points $b_{0}, b_{1} ; c, b_{0} ; b_{1}, c$ in turn. We obtain the result that $a b_{0} b_{1}$ or $b_{1} b_{0} b$ and $a c b_{0}$ or $b_{0} c b$ and $a b_{1} c$ or $c b_{1} b$ exist. But it is readily verified that no one of the eight possible combinations can exist, for assuming any one of them leads, by an application of the transitive property of the betweenness relation in metric spaces, to the conclusion that the triple $b_{0}, c, b_{1}$ is linear, in violation of the relations (1) above. ${ }^{*}$ This completes the proof of the theorem.

UNIVERSITY OF Missouri

\title{
A TRANSFORMATION ASSOCIATED WITH THE TRISECANTS OF A RATIONAL TWISTED QUINTIC CURVE $\dagger$
}

BY L. A. DYE

1. Introduction. This transformation is generated by the use of a $(1,1)$ correspondence between a pencil of ruled cubic surfaces $|F|$, and their simple directrices which are the trisecants of a rational twisted quintic curve $C_{5}$. All of the cubic surfaces contain $C_{5}$ and have the quadrisecant of $C_{5}$ as a double line $l$. Through a general point $P$ of space passes one $F$ whose simple directrix $r$ determines with $P$ a plane tangent to the ruled surface of trisecants of $C_{5}$ at a point $Q$ on $r$. The line $P Q$ meets $F$ in a residual point $P^{\prime}$ which is the image of $P$ in an involutorial Cremona transformation of order 43 . A special feature of the transformation is the existence of two ruled surfaces whose generators are parasitic lines of the transformation. One of these surfaces is a principal surface, and the other is not.

Other transformations generated by a somewhat similar method have been discussed by the author in recent papers. $\ddagger$

2. The Pencil of Cubic Surfaces. The equation of a pencil of cubic surfaces, parameter $\lambda$, with a double line $l \equiv x_{1}=x_{2}=0$, is

* Erste Untersuchung, loc. cit., p. 78. For example, the combination $a b_{0} b_{1}$, $a c b_{0}, a b_{1} c$ cannot exist since the second and third of these relations imply the existence of $b_{1} c b_{0}$.

$\dagger$ Presented to the Society, December 29, 1936.

$\ddagger$ This Bulletin, vol. 42 (1936), pp. 535-540. Tôhoku Mathematical Journal, vol. 43, (1937), pp. 174-177. 


$$
F(x) \equiv \lambda F^{\prime}(x)+F^{\prime \prime}(x) \equiv x_{1} x_{2}\left(a_{1} x_{1}+a_{2} x_{2}\right)+B x_{3}+C x_{4}=0,
$$

where

$$
\begin{aligned}
& B \equiv b_{11} x_{1}{ }^{2}+b_{12} x_{1} x_{2}+b_{22} x_{2}{ }^{2}, C \equiv c_{11} x_{1}{ }^{2}+c_{12} x_{1} x_{2}+c_{22} x_{2}{ }^{2},
\end{aligned}
$$

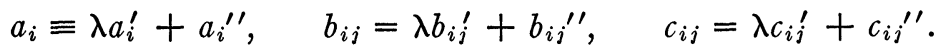

The equations of the simple directrix $r$ of $F(x)$ are

$$
\begin{aligned}
& u \equiv b_{11} D_{1} x_{1}-b_{22} D_{2} x_{2}-D x_{4}=0 \\
& v \equiv c_{11} D_{1} x_{1}-c_{22} D_{2} x_{2}+D x_{3}=0
\end{aligned}
$$

where

$$
\begin{aligned}
D_{i} & \equiv a_{1} A_{1 i}+a_{2} A_{2 i}, \quad D \equiv A_{12}^{2}-A_{11} A_{22}, \\
A_{11} & \equiv b_{12} c_{22}-b_{22} c_{12}, A_{12} \equiv b_{22} c_{11}-b_{11} c_{22}, A_{22} \equiv b_{11} c_{12}-b_{12} c_{11} .
\end{aligned}
$$

If $F(x)$ is multiplied by $D$ it may be factored as follows: $D F(x) \equiv B v-C u$.

Since the line $r$ is a trisecant of $C_{5}$ (the residual intersection of two surfaces $F(x)$ ) the eliminant of $u=v=0$ is the equation of the ruled surface of trisecants, $R$. This surface is of order 8 since $u, v$ are each of order 4 in $\lambda$. The coordinates of a tangent plane to $R$ at any point $(\xi)$ on $r$ are the values at $(\xi)$ of the coefficients of $d x_{i}$ in the form obtained by eliminating $d \lambda$ from the total differentials $d u, d v$. If the plane passes through a general point $P(y)$ of space, the coordinates of the point of contact $Q(z)$ with $R$ are*

$$
\begin{aligned}
& z_{1}=D\left(\gamma_{2} u-\beta_{2} v\right) \\
& z_{2}=D\left(\gamma_{1} u-\beta_{1} v\right) \\
& z_{3}=c_{22} D_{2}\left(\gamma_{1} u-\beta_{1} v\right)-c_{11} D_{1}\left(\gamma_{2} u-\beta_{2} v\right) \\
& z_{4}=-b_{22} D_{2}\left(\gamma_{1} u-\beta_{1} v\right)+b_{11} D_{1}\left(\gamma_{2} u-\beta_{2} v\right)
\end{aligned}
$$

where

$$
\begin{aligned}
\beta_{i} & \equiv D_{i}\left(b_{i i}{ }^{\prime} D^{\prime \prime}-b_{i i}{ }^{\prime} D^{\prime}\right)-D\left(b_{i i}{ }^{\prime} D_{i}{ }^{\prime \prime}-b_{i i}{ }^{\prime} D_{i}^{\prime}\right) \\
\gamma_{i} & \equiv D_{i}\left(c_{i i}{ }^{\prime} D^{\prime \prime}-c_{i i}^{\prime \prime} D^{\prime}\right)-D\left(c_{i i}{ }^{\prime} D_{i}{ }^{\prime \prime}-c_{i i}{ }^{\prime \prime} D_{i}^{\prime}\right) \\
D^{\prime} & \equiv \partial D / \partial \lambda, D^{\prime \prime} \equiv 4 D-\lambda D^{\prime}, D_{i}^{\prime} \equiv \partial D_{i} / \partial \lambda, D_{i}^{\prime \prime} \equiv 3 D_{i}-\lambda D_{i}^{\prime}
\end{aligned}
$$

* From here on the variable $y_{i}$ will replace $x_{i}$ in all forms and no change in notation will be made. 
The residual intersection of the line $P Q$ with the surface $F$ is the point $P^{\prime}\left(y^{\prime}\right)$ whose coordinates are

$$
y_{i}^{\prime}=y_{i}\left(y_{i} \partial F(z) / \partial z_{i}\right)-z_{i}\left(z_{i} \partial F(y) / \partial y_{i}\right) .
$$

3. An Involution on $l$. Any plane through $r$ cuts two residual lines from a surface $F$ which with $l$ determine a conjugate pair of planes in an involution $I$ on $l$. The equations of this involution are

$$
y_{1}^{\prime}=A_{12} y_{1}-A_{11} y_{2}, \quad y_{2}^{\prime}=A_{22} y_{1}-A_{12} y_{2} .
$$

The planes determined by $l$ and the two torsal generators of $F$ are the double planes of $I$. They are

$$
K \equiv A_{22} y_{1}^{2}-2 A_{12} y_{1} y_{2}+A_{11} y_{2}^{2}=0 .
$$

The three planes through $l$ and the tangents to $C_{5}$ at its three points on $r$ are

$$
D T \equiv y_{2}\left(B \gamma_{2}-C \beta_{2}\right)-y_{1}\left(B \gamma_{1}-C \beta_{1}\right)=0 .
$$

The associated triad of planes in $I$ is

$$
\begin{aligned}
L \equiv\left(A_{22} y_{1}-A_{12} y_{2}\right)\left(B \gamma_{2}-\right. & \left.C \beta_{2}\right) \\
& -\left(A_{12} y_{1}-A_{11} y_{2}\right)\left(B \gamma_{1}-C \beta_{1}\right) .
\end{aligned}
$$

The involution fails when $D=0$. The four cubic surfaces which correspond to these values of $\lambda$ are the Cayley surfaces of the pencil, that is, they are surfaces in which the simple directrix $r$ is adjacent to $l$, and along $l$ there is a fixed tangent plane to each surface.

4. The Involutorial Space Transformation. If $\lambda$ is replaced by its value $-F^{\prime \prime}(y) / F^{\prime}(y)$, in the equations defining $\left(y^{\prime}\right)$, these become the equations of a space transformation.* The forms $u, v$ are now factorable into $B R, C R$ respectively, and the polars of $F$ factor as follows: $y_{i} \partial F(z) / \partial z_{i} \equiv T D^{3} R^{3} L$, and $z_{i} \partial F(y) / \partial y_{i}$ $\equiv T D^{2} R^{2} K$. This results in the factors $T D^{2} R^{3}$ coming off from the transformation, and its equations now take the form

* In order to avoid a confusing number of new symbols the same letters used heretofore to represent functions of $(\lambda, y)$ will now be used to represent the same functions after the substitution for $\lambda$, 


$$
\begin{aligned}
& y_{1}^{\prime}=\left(A_{12} y_{1}-A_{11} y_{2}\right) T D^{2}, \\
& y_{2}^{\prime}=\left(A_{22} y_{1}-A_{12} y_{2}\right) T D^{2}, \\
& y_{3}^{\prime}=v L-T D\left[c_{11} D_{1}\left(A_{12} y_{1}-A_{11} y_{2}\right)-c_{22} D_{2}\left(A_{22} y_{1}-A_{12} y_{1}\right)\right], \\
& y_{4}^{\prime}=-u L+T D\left[b_{11} D_{1}\left(A_{12} y_{1}-A_{11} y_{2}\right)\right. \\
&\left.\quad-b_{22} D_{2}\left(A_{22} y_{1}-A_{12} y_{1}\right)\right] .
\end{aligned}
$$

It is seen from the equations of the transformation that the four Cayley surfaces taken twice, and the surface $T$ of order twelve, are the images of the line $l$. Geometrically this can be explained in the following manner. Any plane through $l$ cuts a Cayley surface in a generator $s$. The point of contact $Q$ of the plane with $R$ is on $l$, and each point of $s \sim Q$ or $Q \sim s$. The surface $T$ is generated by the tangents to $C_{5}$ at points on a trisecant $r$. Such a tangent line passes through the point of contact $Q$ of the tangent plane to $R$, so that each point of the line corresponds to the whole line. The line is parasitic and the surface $T$ is a factor of the transformation, but the tangent line meets $l$ and the image of the point on $l$ is the tangent line and, as the image of $l$, the surface $T$ stays in the transformation as a principal surface.

The plane tangent to $R$ at a point on $C_{5}$ on a trisecant $r$ cuts a residual line from $F$ in addition to the trisecant and the tangent. These residual lines are the images of the points on $C_{5}$. They generate the surface $L$ of order 30 . In any plane through $r$ the points of $C_{5}$ not on $r$ go into points on the two secants cut from $F$. These image points generate a surface $G$ of order 24 which is an additional image of $C_{5}$. The form of this surface is most easily obtained by subjecting an $F$ to the transformation $F \sim 4 D+2 T+L+G+F$.

When a plane through $r$ passes through a torsal generator of $F$, then each point of the generator corresponds to itself, since the lines joining such points to the point of contact $Q$ are tangent to $F$. These tangent lines generate the invariant octavic surface, $K$.

Each generator of $T$ determines two generators of $K$, and each generator of $K$ determines three generators of $T$. In this $(2,3)$ correspondence there are five coincidences, that is, there are five torsal generators of surfaces $F$ which are tangent to $C_{5}$ at a 
point on the trisecant of the surface. These five lines $l_{i}$ are parasitic double lines on the homoloidal surfaces.

The only remaining fundamental lines are the trisecants of $C_{5}$. These lines are all parasitic and the surface $R$ is shed off the transformation and does not appear as a principal surface.

5. Table of Characteristics. The following table exhibits the nature of the images of the several fundamental elements of the transformation.

$$
\begin{aligned}
S_{1} \sim & S_{43}: l^{30} 4 \bar{l}^{3} C_{5}^{13+t} 5 l_{i}^{2}, \\
l \sim & 2 D_{12}: l^{8} 4 \bar{l} C_{5}^{4}+T_{12}: l^{9} C_{5}^{3+t} 5 l_{i}, \\
C_{5} \sim & l^{22} 4 \bar{l} C_{5}^{9} 5 l_{i}+G_{24}: l^{16} 4 \bar{l}^{4} C_{5}^{7+t} 5 l_{i}^{3}, \\
K_{8} \sim & K_{8}: l^{6} 4 \bar{l} C_{5}^{2} 5 l_{i}, \\
& R_{8}: l^{4} C_{5}^{3+t} 5 l_{i} .
\end{aligned}
$$

The notation $4 \bar{l}$ is used to indicate that four sheets of the surface have contact along $l$ with the four Cayley surfaces. The notation $C_{5}{ }^{t}$ denotes contact along $C_{5}$ due to the fact that $R$ and $T$ have the same tangent planes at each point of $C_{5}$. In the total intersection of $K$ and $S_{43}$ the line $l$ must be counted an additional four times since it plays the role of an invariant line in the four fixed tangent planes to the Cayley surfaces. The complete intersection of two homoloidal surfaces may be set down as follows

$$
\left[S_{43}, S_{43}^{\prime}\right]=l^{900}+4 \bar{l}^{9}+C_{5}^{169+1}+5 l_{i}^{4}+C_{43} .
$$

The Citadel 